\title{
Apoio Social e Comportamentos de Risco na Adolescência
}

\author{
Cássia Ferrazza Alves \\ Débora Dalbosco Dell’Aglio \\ Universidade Federal do Rio Grande do Sul \\ Porto Alegre, RS, Brasil
}

\begin{abstract}
RESUMO
O apoio social tem sido considerado fator de proteção ao engajamento de adolescentes em comportamentos de risco. Este estudo investigou a relação entre apoio social e comportamentos de risco (comportamentos sexual, infracional, suicida e uso de substâncias) em 374 adolescentes de Porto Alegre, entre 12 e 18 anos, de escolas públicas. Os instrumentos utilizados foram uma escala de apoio social e questões que avaliaram comportamentos de risco. Análises foram realizadas com teste $t$ de Student, Correlações de Pearson e Análise de Regressão Múltipla. Os resultados indicaram maior envolvimento em comportamentos de risco em adolescentes mais velhos e diferenças por sexo (meninos envolveram-se mais em comportamento infracional e sexual de risco e as meninas em comportamento suicida). O apoio da família e dos professores esteve associado ao menor envolvimento nesses comportamentos e o apoio dos amigos ao maior engajamento. Estratégias de prevenção e intervenção direcionadas aos jovens devem considerar a influência dessas variáveis na adesão aos comportamentos de risco.
\end{abstract}

Palavras-chave: Adolescência; Apoio Social; Comportamento de Risco.

\section{ABSTRACT}

Social Support and Risk Behavior in Adolescence

Social support can be considered a protective factor as regards engagement of the adolescent in risk behaviors. This study investigated the relation between social support and risk behaviors (sexual behaviors, criminal, suicidal and substance use) in adolescents in three-hundred and seventy-four adolescents from Porto Alegre, from 12 to 18 years old, from public schools. Social support scale and questions about risk behavior were used. Student's t-Test, Pearson's Correlation and Multiple Regression Analysis were conducted. More engagement in risk behaviors in older adolescents was evidenced, as well as differences by sex (boys were more engaged in criminal behavior and risk sexual behavior and the girls in suicidal behavior). Support from the family and teachers was perceived as a factor associated to lower engagament in these behaviors and the support from friends was associated to higher engagement. Strategies of prevention and intervention directed to young people should consider the influence of these variables regarding adherence to risk behaviors.

Keywords: Adolescence; Social Support; Risk Behavior.

\section{RESUMEN}

\section{Apoyo Social y Comportamientos de Riesgo en la Adolescencia}

El apoyo social ha sido considerado factor de protección a la participación del adolescente en comportamientos de riesgo. Este estudio investigó la relación entre apoyo social y comportamientos de riesgo (comportamientos sexuales, infractor, suicida y uso de substancias) en 374 adolescentes de Porto Alegre/RS, entre 12 y 18 años, de escuelas públicas. Los instrumentos fueron una escala de apoyo social y las preguntas que evaluaron los comportamientos de riesgo. Análisis se realizaron con teste $t$ de Student, Correlación de Pearson y Análisis de Regresión Múltiple. Los resultados indicaron mayor implicación en comportamientos de riesgo en adolescentes más viejos y diferencias por sexo (niños se envolvieron más en infracciones y conductas sexuales de riesgo y las niñas en comportamiento suicida). El apoyo de la familia y de los profesores estuvo asociado a la menor implicación en estos comportamientos de riesgo y el apoyo de los amigos para una mayor participación. Estrategias de prevención e intervención direccionadas a los jóvenes deben considerar la influencia de estas variables en la adhesión a los comportamientos de riesgo.

Palabras clave: Adolescencia; Apoyo Social; Comportamiento de Riesgo. 


\section{INTRODUÇÃO}

Comportamentos de risco são comportamentos que podem ser considerados como potencialmente capazes de ameaçar à saúde e reduzir a qualidade de vida do indivíduo, colocando-o em risco (Brener et al., 2013). Atualmente, diversos comportamentos de risco têm sido descritos na literatura, tais como envolvimento em brigas, agressão e atos infracionais (Câmara, Sarriera, \& Carlotto, 2007; Centers for Disease Control and Prevention, 2012; Farias Júnior et al., 2009), uso de substâncias (álcool, cigarro, drogas) (Costa et al., 2013, Dumas, Ellis, \& Wolfe, 2012; Farias Júnior et al., 2009; Locatelli et al., 2011) comportamento sexual de risco (Câmara et al., 2007; Cruzeiro et al., 2010; Farias Júnior et al., 2009), comportamento suicida (Braga \& Dell'Aglio, 2013; Centers for Disease Control and Prevention, 2012; Kim, Moon \& Kim, 2011), alimentação não saudável (Farias Júnior et al., 2009) ausência de prática de atividade física (Farias Júnior et al., 2009) e comportamento de risco no trânsito (Câmara et al., 2007), entre outros. O comportamento de risco tem sido associado à adolescência, pois essa etapa é percebida como um período que envolve transformações físicas e sociais, busca de autonomia e o contato com novas experiências, podendo ser considerada como um período de maior vulnerabilidade ao risco. Os estudos evidenciam, ainda, que o envolvimento em comportamentos de risco pode ser mediado pelo apoio social, o qual pode configurar-se como fator de proteção ou de risco, dependendo das características dos contextos de inserção e das redes de apoio do adolescente (Springer, Parcel, Baumler \& Ross, 2006).

\section{COMPORTAMENTOS DE RISCO}

A relação entre comportamentos de risco e adolescência é mediada por questões biológicas, sociais e contextuais. Considerando o ponto de vista de Steinberg (2004), os comportamentos de risco são esperados durante a adolescência e considerados como um processo biologicamente dirigido e normativo, pois há, ao mesmo tempo, o aumento de novidades e busca de sensações durante a puberdade e um imaturo sistema de autorregulação, que tem por objetivo modular a busca por recompensas e previsão de ações (Steinberg, 2004). Diante disso, a maturação do sistema de controle cognitivo, responsável pelo controle dos impulsos, é mais lenta e muitos adolescentes podem agir impulsivamente, não refletindo sobre as consequências de seus atos (Steinberg, 2007).
Em contraposição ao ponto de vista da neurociência, alguns autores procuram destacar que os adolescentes não são os únicos que se envolvem em riscos e atribuem o envolvimento em comportamentos de risco a influências sociais e contextuais, além de buscar conhecer como esses fatores mediam a relação entre a adolescência e tais atitudes (Males, 2010; Willoughby, Good, Adachi, Hamza \& Tavernier, 2013). Além disso, é preciso compreender que o envolvimento em comportamentos de risco também acontece em outras etapas do ciclo vital (Willoughby et al., 2013) e que o contexto social regula a tomada de decisão frente aos comportamentos de risco (Albert \& Steinberg, 2011; Steinberg, 2004).

Diante do exposto, torna-se um desafio diferenciar um comportamento típico da adolescência de um comportamento que pode acarretar maiores prejuízos no desenvolvimento dos jovens. Lerner e Galambos (1998) destacam alguns aspectos que podem trazer maior comprometimento à saúde do adolescente: (1) envolvimento precoce; (2) engajamento continuado como, por exemplo, quando o adolescente ingere álcool continuamente; e (3) manifestação de comportamentos de risco como estilo de vida, aderindo a vários comportamentos de risco simultaneamente.

Quanto ao envolvimento de adolescentes brasileiros nos comportamentos de risco abordados nesse estudo, no estudo de Cruzeiro et al. (2010) foi observado que $53,4 \%$ dos jovens já haviam tido sua primeira relação sexual, sendo a maioria meninos. Apenas $56,3 \%$ usaram preservativo nas últimas três relações sexuais. As meninas apresentaram maior tendência para comportamento sexual de risco, considerando a frequência do uso de preservativos nas últimas três relações sexuais.

Ao investigar uso de substâncias, os estudos nacionais e internacionais revelam que grande parte dos adolescentes já usou álcool, cigarro e outras drogas. No estudo de Costa et al. (2013), com jovens brasileiros de 10 a 17 anos, 31,1\% já experimentaram álcool e 5,9\% apresentavam uso problemático, como ingerir a substância com mais frequência, sendo a maioria meninos e mais velhos. Ao avaliar envolvimento em atos infracionais, os autores investigam o envolvimento em brigas, uso ou o fato de carregar arma de fogo ou arma branca (como faca). 34,1\% de adolescentes investigados no estudo de Brookmeyer, Henrich, Cohen e Shahar (2010) já relataram o envolvimento em pelo menos um comportamento infracional, enquanto que $12,7 \%$ já relataram o envolvimento em dois ou mais comportamentos. Estudo como o de Farias Júnior et al. (2009) encontrou diferenças por sexo, sendo 
que os meninos apresentam maior envolvimento do que as meninas em brigas.

Quanto ao comportamento suicida, levantamento realizado por Abasse, Oliveira, Silva e Souza (2009), sobre o número de internações realizadas no SUS do estado de Minas Gerais por tentativa de suicídio, encontrou que cerca de $16 \%$ da amostra eram jovens entre 10 e 19 anos, denotando a necessidade de atenção a esse risco nesse período do ciclo vital. Diferenças por sexo têm sido observadas, sendo as tentativas de suicídio mais frequentes entre as meninas e o suicídio propriamente dito mais presente entre os meninos. Além disso, fatores de risco como transtornos psicológicos, uso de substâncias, exposição à violência e comportamento sexual de risco podem estar associados a esse comportamento (Braga \& Dell'Aglio, 2013; Kim et al., 2011).

Nesse sentido, muitos estudos destacam o envolvimento dos jovens em mais de um comportamento de risco. O consumo de álcool e de drogas em adolescentes brasileiros (Castro, Cunha, \& Souza, 2011) esteve relacionado a comportamentos violentos. Os jovens que apresentaram comportamento violento apresentaram também mais comportamentos sexuais de risco como o não uso de preservativos. Da mesma forma, adolescentes usuários de drogas estiveram mais envolvidos com tráfico de drogas, problemas com a justiça e prisões do que os não usuários (Nardi, Cunha, Bizarro, \& Dell'Aglio, 2012). No questionário nacional sobre comportamentos de risco em estudantes nos Estados Unidos, considerando a última relação sexual, $22,1 \%$ ingeriu álcool ou usou outras drogas e $12,9 \%$ não utilizou nenhum método contraceptivo (Centers for Disease Control and Prevention, 2012). Assim como nos adolescentes americanos, em um estudo com adolescentes brasileiros (Câmara et al., 2007), aqueles que apresentaram mais parceiros sexuais no último ano e maior frequência no uso de álcool, apresentaram maior tendência a comportamento sexual de risco, como o não uso de preservativo.

\section{APOIO SOCIAL}

O apoio social é definido, segundo Cobb (1976), como o conjunto de informações que levam um indivíduo a acreditar que é estimado, amado, cuidado e pertencente a uma rede com obrigações mútuas. Ao sentir que pode ser apoiado por um grupo de pessoas, o indivíduo tende a estar mais protegido nas situações de crises, facilitando as estratégias de enfrentamento e adaptação às mudanças (Cobb, 1976).

$\mathrm{Na}$ literatura brasileira, os estudos que investigam apoio social e adolescência têm sido realizados em diferentes contextos envolvendo, por exemplo, a rede de apoio de pais adolescentes (Levandowski, Barth, Munhós, Rodde, \& Wendland, 2012), adolescentes com doenças crônicas (Araújo, Neusa, Gomes, \& Nóbrega, 2011), em situação de institucionalização (Siqueira, Tubino, Schwarz, \& Dell'Aglio, 2009) e em risco psicossocial (Amparo, Galvão, Cardenas, \& Koller, 2008), entre outros. Em geral, os estudos indicam a presença de, pelo menos, três fontes de apoio importantes para o desenvolvimento do adolescente: 0 apoio da família, da escola e dos amigos.

A família é considerada a primeira e maior fonte de apoio social na vida do indivíduo, pois é no espaço familiar que a maioria das pessoas aprende a receber e a oferecer apoio (Burleson \& Kunkel, 1996; Squassoni, 2009). Além disso, o apoio dos pais tem sido relacionado a menos envolvimento em comportamentos de risco (Fosco, Stormshak, Dishion, \& Winter, 2012; Springer et al., 2006), menos sintomas depressivos (Ellis, Nixon, \& Williamson, 2009; Weber, Puskar, \& Ren, 2010) e maior engajamento na escola, juntamente com o apoio dos professores (Wang \& Eccles, 2012).

A escola é outro importante contexto de apoio social em que os adolescentes passam grande parte do seu tempo. Pode ser considerada como um ambiente que influencia na autoestima, na formação cidadã e na saúde mental, sendo que e o apoio dos professores está relacionado a menos problemas de comportamento (Amparo et al., 2008; Rueger, Malecki, \& Demaray, 2010; Wang \& Dishion, 2011). Tem sido observado que o ambiente escolar e seus colaboradores, como professores e equipe diretiva, são percebidos pelos jovens como fontes de confiança, embora nem sempre isso aconteça como demonstra a pesquisa de Amparo et al. (2008). Nessa pesquisa, a maioria dos adolescentes declarou que gostava dos professores, porém esse índice diminuiu quando se referia à possibilidade de poder ter confiança nos educadores.

Outra fonte importante de apoio social dos adolescentes são os amigos, pois a adolescência é um período de busca por novas formas de identificação, sendo esperado que os jovens busquem outros vínculos sociais fora do ambiente familiar. Esse apoio tem sido evidenciado tanto como fator de proteção, influenciando na autoestima e autoeficácia, e diminuindo sintomas depressivos (Auerbach, Bigda-Peyton, Ebarhart, Webb, \& Ringo Ho, 2011; Ellis et al., 2009) quanto como fator de risco. Como fator de risco, a influência dos pares pode estar associada ao envolvimento em comportamentos de risco, tais como comportamentos sexuais de risco, uso de substâncias e comportamento infracional (Choo \& Sim, 2010; Dumas et al., 2012; Michael \& BemZur, 2007). No entanto, os estudos demonstram que 
há diferenças nos resultados dependendo do tipo de apoio dos amigos. No estudo de Wang e Eccles (2012), os adolescentes com baixo envolvimento com a escola tinham amigos com algumas condutas antissociais e os jovens que estavam mais envolvidos positivamente com a escola tinham amigos com características prósociais. A influência negativa dos amigos foi mediada pela influência positiva do apoio dos adultos, sugerindo que adolescentes que apresentaram baixo envolvimento com a escola apresentaram, ao mesmo tempo, baixo apoio dos pais e professores combinado com alto apoio dos pares com características antissociais. Logo, é importante que os jovens possam contar com diferentes fontes de apoio (adultos e amigos) ao longo do seu desenvolvimento.

Ao relacionar apoio social e comportamento de risco envolvendo jovens brasileiros, Raffaelli, Koller e Cerqueira-Santos (2012) encontraram que os adolescentes em situação de vulnerabilidade social que apresentavam altos níveis de fatores de proteção (apoio da família e escola, percepção de autoeficácia) apresentaram menos envolvimento em uso de substâncias, comportamento sexual de risco e comportamento infracional comparados a adolescentes com poucos fatores de proteção. Também, no estudo de Costa (2009), o apoio da família, da escola e da comunidade foi protetivo para o uso de drogas entre jovens brasileiros de 14 a 24 anos.

A partir disso, este estudo teve por objetivo verificar a relação entre apoio social e comportamentos de risco em adolescentes da cidade de Porto Alegre/RS. Para isso, foram analisados simultaneamente os seguintes comportamentos de risco: (1) comportamento sexual de risco; (2) uso de substâncias (álcool, cigarro e outras substâncias); (3) comportamento infracional; e (4) comportamento suicida. O apoio social será analisado a partir dos seguintes tipos de apoio percebido: apoio da família, dos professores, dos amigos e da comunidade em geral.

\section{MÉTODO}

\section{Participantes}

Participaram 374 adolescentes com idades entre 12 e 18 anos $(M=14,73 ; S D=1,41)$, estudantes entre a $6^{\underline{a}}$ série do ensino fundamental e $3^{\mathrm{o}}$ ano do ensino médio de escolas públicas da cidade de Porto Alegre/ RS. $66,3 \%$ eram do sexo feminino e $33,7 \%$ do sexo masculino, e moravam com suas famílias.

\section{Instrumentos}

Foi utilizado o Questionário da Juventude Brasileira (QJB, Dell'Aglio, Koller, Cerqueira-Santos,
\& Colaço, 2011), composto por 77 questões que avaliam fatores de risco e proteção. Para este estudo foram utilizados os dados sociodemográficos e as questões referentes a comportamentos sexuais, uso de substâncias, envolvimento em atos infracionais e comportamento suicida. As questões contidas nesse questionário formaram o Índice de Comportamentos de Risco (ICR), que tinha por objetivo criar uma pontuação de comportamentos de risco (Alves, Zappe, \& Dell'Aglio, no prelo). Esse Índice avalia quatro tipos de comportamentos de risco: comportamento sexual (quatro questões: idade da primeira relação sexual, número de parceiros sexuais no último ano, frequência de uso de preservativo e uso de métodos contraceptivos), uso de substâncias como álcool, cigarro e drogas ilícitas (oito questões: experimentação de substância, idade de início de uso das substâncias citadas, frequência de uso, parceiro do uso de drogas), comportamento infracional (duas questões: envolvimento em situações ilegais como brigas, pichação, destruição de propriedade, assalto e roubo; e número de situações ilegais que o jovem se envolveu) e comportamento suicida (três questões: presença ou não de ideação e tentativa de suicídio, frequência de ideação e tentativa de suicídio). O ICR apresenta 17 itens com pontuação que pode variar entre zero e 34 pontos. Cada item avalia a ausência de risco (zero), baixo risco (um ponto) e alto risco (dois pontos). $\mathrm{O}$ escore do participante é somado a partir do tipo de risco para cada um dos quatro tipos de comportamento. O escore total também é somado. A construção do ICR foi baseada em estudos nacionais e internacionais que avaliaram comportamentos de risco em adolescentes e a busca por elaborar um índice que avaliasse simultaneamente vários comportamentos de risco. O estudo original apresentou uma Alpha de Cronbach de 0,84, e foi realizada uma Análise de Componentes Principais, método Varimax, confirmando os fatores estabelecidos a priori (Alves et al., no prelo).

Para conhecer a percepção de apoio dos adolescentes, foi utilizada a Social Support Appraisals (SSA; Vaux et al., 1986), adaptada para o Brasil por Squassoni e Matsukura (2014). É uma escala likert de seis pontos que variam de "concordo totalmente" a "discordo totalmente", composta por quatro subescalas: amigos, família, professores e apoio geral. Apresenta sete questões negativas e 23 questões afirmativas, tais como "eu sou bastante querido pela minha família", "eu me sinto muito ligado aos meus amigos". Os valores na escala variam entre 30 e 180 e o estudo original apresentou boa consistência interna, com Alpha de Cronbach de 0,74 (Squassoni \& Matsukura, 2014). 
Para as análises com o SSA (Squassoni \& Matsukura, 2014), os itens positivos foram invertidos, a fim de que quanto maior a percepção de apoio, maior fosse o escore na escala. Foi computado um escore total da escala, assim como um escore em cada subescala de apoio social.

\section{Procedimentos e considerações éticas}

A pesquisa foi aprovada pelo Comitê de Ética em Pesquisa do Instituto de Psicologia da UFRGS (parecer 2009060). Foi realizado cálculo amostral (Barbetta, 2001) com margem de erro de $4 \%$, considerando o número de alunos matriculados no ensino público entre a $7^{\underline{a}}$ série do ensino fundamental e o $3^{\mathrm{o}}$ ano do ensino médio. Foi composta uma amostra aleatória por conglomerados, a partir do sorteio das escolas participantes (12 escolas estaduais e uma municipal) e das turmas. Em média, participaram 50 adolescentes por escola, cujos pais assinaram o Termo de Consentimento Livre e Esclarecido.

Foram realizadas duas etapas de coleta de dados nas escolas, com intervalo médio de 10 meses. Na primeira etapa, foi aplicado o QJB (Dell'Aglio et al., 2011), com uma amostra de 689 adolescentes. Na segunda etapa, foi aplicado o SSA (Vaux et al., 1986; Squassoni \& Matsukura, 2014) em amostra de 376 adolescentes, com perda amostral de $46 \%$. A aplicação dos questionários nos dois momentos foi feita coletivamente, tendo duração máxima de 60 minutos.

\section{Análise dos dados}

Foram realizadas análises descritivas através de médias e desvio-padrão, e análises inferenciais através do teste $t$ de Student, correlações de Pearson e Análise de Regressão Múltipla, método Stepwise. Foi utilizado como variável dependente o escore de comportamento de risco computado a partir do ICR e como variáveis independentes os escores de apoio social, considerando o tipo de apoio e a idade.

\section{RESULTADOS}

Na Tabela 1, estão descritas as médias e os desviospadrão por tipo de comportamento de risco e sexo. Foi encontrada diferença estatisticamente significativa no comportamento sexual de risco $(t=3,55 ; g l=178,77$; $p<0,01)$, comportamento infracional $(t=3,89$; $g l=170,07 ; p<0,01)$ e comportamento de risco total $(t=1,96 ; g l=372 ; p=0,05)$, sendo que os meninos apresentaram médias superiores às meninas. Em contrapartida, as meninas apresentaram médias superiores no comportamento suicida $(t=-2,45$; $g l=318,32 ; p=0,015)$ em relação aos meninos.
TABELA 1

Médias e Desvio-Padrão dos Tipos de Comportamento de Risco por Sexo

\begin{tabular}{lccc}
\hline & $\begin{array}{c}\text { Meninos } \\
(n=123)\end{array}$ & $\begin{array}{c}\text { Meninas } \\
(n=251)\end{array}$ & $t$ \\
\hline $\begin{array}{l}\text { Comportamento sexual } \\
\text { de risco }\end{array}$ & $1,47( \pm 2,02)$ & $0,76( \pm 1,37)$ & $3,55^{* *}$ \\
$\begin{array}{l}\text { Uso de álcool, cigarro e } \\
\text { outras substâncias }\end{array}$ & $3,61( \pm 3,10)$ & $3,34( \pm 2,96)$ & 0,82 \\
$\begin{array}{l}\text { Comportamento } \\
\text { infracional }\end{array}$ & $0,77( \pm 1,31)$ & $0,27( \pm 0,82)$ & $3,89^{* *}$ \\
$\begin{array}{l}\text { Comportamento suicida } \\
\text { ICR Total }\end{array}$ & $0,48( \pm 1,23)$ & $0,86( \pm 1,67)$ & $-2,45^{*}$ \\
\hline
\end{tabular}

${ }^{*} p<0,05 *{ }^{*} p<0,01$

Na Tabela 2, estão descritos os tipos de apoio percebido por meninos e meninas. Foi encontrada diferença estatisticamente significativa na percepção de apoio dos amigos $(t=-1,99 ; g l=372 ; p=0,047)$ e no apoio total $(t=-1,99 ; g l=372 ; p=0,047)$, com média mais alta entre as meninas.

TABELA 2

Médias e Desvio-Padrão da Percepção de Apoio Social

\begin{tabular}{lccc}
\hline & $\begin{array}{c}\text { Meninos } \\
(n=123)\end{array}$ & $\begin{array}{c}\text { Meninas } \\
(n=251)\end{array}$ & $t$ \\
\hline Apoio família & $42,11( \pm 6,14)$ & $42,61( \pm 6,59)$ & $-0,72$ \\
Apoio professores & $29,71( \pm 6,87)$ & $30,95( \pm 6,32)$ & $-1,73$ \\
Apoio amigos & $35,02( \pm 5,86)$ & $36,24( \pm 5,47)$ & $-1,99 *$ \\
Apoio geral & $38,48( \pm 6,03)$ & $39,70( \pm 6,31)$ & $-1,78$ \\
Total apoio & $145,31( \pm 19,17)$ & $149,51( \pm 19,11)$ & $-1,99 *$ \\
$* p<0,05$ & & &
\end{tabular}

Para observar a relação entre as variáveis de percepção de apoio, idade e tipos de comportamento de risco, foram realizadas Correlações de Pearson, descritas na Tabela 3. Verifica-se que o apoio da família, dos professores e apoio geral correlacionaramse negativamente com os comportamentos de risco e a idade correlacionou-se positivamente com alguns comportamentos de risco.

Para conhecer as variáveis associadas ao comportamento de risco, foi realizada Análise de Regressão Múltipla, método Stepwise forward. No step 1 foi incluída a variável idade; no segundo modelo (step 2), a idade e apoio da família; no step 3, idade, apoio dos amigos e da família; e no step 4 idade, apoio da família, dos amigos e professores. Na Tabela 4 estão apresentadas as variáveis que foram significativas para o modelo, que explicou $14 \%$ da variação do comportamento de risco. 
TABELA 3

Correlações de Pearson entre Variáveis Estudadas

\begin{tabular}{lcccccc}
\hline & Idade & $\begin{array}{c}\text { Comportamento } \\
\text { sexual }\end{array}$ & $\begin{array}{c}\text { Uso de } \\
\text { substancias }\end{array}$ & $\begin{array}{c}\text { Comportamento } \\
\text { infracional }\end{array}$ & $\begin{array}{c}\text { Comportamento } \\
\text { suicida }\end{array}$ & Risco total \\
\hline Apoio família & 0,01 & $-0,04$ & $-0,17^{* *}$ & $-0,13^{* *}$ & $-0,26^{* *}$ & $-0,21^{* *}$ \\
Apoio prof. & $-0,04$ & $-0,11^{*}$ & $-0,12^{*}$ & $-0,15^{* *}$ & $-0,08$ & $-0,16^{* *}$ \\
Apoio amig. & $-0,14^{* *}$ & $-0,03$ & 0,02 & 0,01 & $-0,07$ & $-0,02$ \\
Apoio geral & 0,01 & 0,02 & $-0,07$ & $-0,04$ & $-0,18^{* *}$ & $-0,09$ \\
Total apoio & $-0,05$ & $-0,05$ & $-0,11^{*}$ & $-0,20^{* *}$ & $-0,16^{* *}$ \\
Idade & 1 & $0,25^{* *}$ & $0,26^{* *}$ & 0,07 & $-0,01$ & $0,24^{* *}$ \\
\hline
\end{tabular}

$* p<0,05 ; * * p<0,01$

TABELA 4

Análise de Regressão Múltipla

\begin{tabular}{|c|c|c|c|c|c|}
\hline \multirow{3}{*}{ Itens } & \multicolumn{5}{|c|}{ Comportamento de risco } \\
\hline & \multicolumn{2}{|c|}{ Coeficientes não padronizados } & \multicolumn{3}{|c|}{ Coeficientes padronizados } \\
\hline & $B$ & StdError & Beta & $t$ & 95 I.C. para $B$ \\
\hline \multicolumn{6}{|l|}{ Step 1} \\
\hline Idade & 0,95 & 0,18 & 0,26 & 5,24 & {$[0,60 ; 1,31]$} \\
\hline \multicolumn{6}{|l|}{ Step 2} \\
\hline Idade & 0,95 & 0,18 & 0,26 & 5,35 & {$[0,60 ; 1,30]$} \\
\hline Apoio família & $-0,17$ & 0,04 & $-0,21$ & $-4,38$ & {$[-0,25 ;-0,09]$} \\
\hline \multicolumn{6}{|l|}{ Step 3} \\
\hline Idade & 1,04 & 0,18 & 0,28 & 5,84 & {$[0,70 ; 1,40]$} \\
\hline Apoio família & $-0,23$ & 0,04 & $-0,28$ & $-5,24$ & {$[-0,31 ;-0,14]$} \\
\hline Apoio amigos & 0,14 & 0,05 & 0,16 & 2,87 & {$[0,04 ; 0,24]$} \\
\hline \multicolumn{6}{|l|}{ Step 4} \\
\hline Idade & 1,04 & 0,18 & 0,29 & 5,41 & {$[0,69 ; 1,39]$} \\
\hline Apoio família & $-0,20$ & 0,04 & $-0,26$ & $-4,64$ & {$[-0,29 ;-0,12]$} \\
\hline Apoio amigos & 0,17 & 0,05 & 0,18 & 3,27 & {$[0,07 ; 0,27]$} \\
\hline Apoio professores & $-0,09$ & 0,04 & $-0,12$ & $-2,38$ & {$[-0,18 ;-0,02]$} \\
\hline $\mathrm{R}^{2}=0,14$ & & & & & \\
\hline
\end{tabular}

\section{DISCUSSÃO}

No conjunto, os resultados que merecem destaque referem-se à idade, ao apoio da família, dos professores e amigos, e às diferenças por sexo no envolvimento em comportamentos de risco e percepção de apoio social. Quanto à idade, as correlações positivas com comportamento sexual de risco, uso de substâncias e comportamento de risco total, além da Análise de Regressão, indicam que há uma tendência no sentido de que, em adolescentes mais velhos, haja maior envolvimento em comportamentos de risco. Essa tendência também foi observada nos estudos de Costa et al. (2013), Farias Júnior et al. (2009), e Locatelli et al. (2011), demonstrando que o envolvimento em riscos apresenta variação ao longo da adolescência. White e Jackson (2005) ressaltam que, no período de transição entre a adolescência e a idade adulta, o monitoramento parental decresce e o jovem tem maior liberdade frente às regras impostas pelos adultos, exercitando sua autonomia. Também ocorre o afastamento da família e maior aproximação ao grupo de iguais (Wagner, Falcke, Silveira, \& Mosmann, 2002).

Neste estudo, o apoio dos amigos mostrou-se associado ao comportamento de risco, sendo que maior apoio dos amigos foi associado a maior comportamento 
de risco como encontrado em outros estudos (Choo \& Sim, 2010; Dumas et al., 2012). A literatura tem evidenciado que os pares se constituem em um estímulo influente no processo de tomada de decisões que envolvam algum risco, pois quando o adolescente está junto com os amigos pode tomar decisões de maior risco do que quando está sozinho (Albert, Chein, \& Steinberg, 2013). Wang e Eccles (2012) também destacam que amigos com condutas antissociais podem estar mais relacionados ao aumento da manifestação de comportamentos de risco. Seria interessante conhecer de forma mais ampla as características dos grupos de amigos que os jovens se envolvem. Em contrapartida ao apoio dos pares, o apoio da família correlacionou-se negativamente com uso de substâncias, comportamento infracional, suicida e comportamento de risco total. $\mathrm{Na}$ Regressão Linear, o apoio da família apresentou maior associação com comportamento de risco, demonstrando que o adolescente que percebe que é amado, querido e respeitado pela família pode apresentar menor envolvimento nesses comportamentos. Esse resultado também foi observado nos estudos de Auerbach et al. (2011), Fosco et al. (2012), Rueger et al. (2010), Springer et al. (2006) e Weber et al. (2010). Nesse sentido, considerando que a adolescência é compreendida como uma etapa de muitas mudanças na vida do jovem, é importante que o contexto familiar adaptese a esse novo momento, buscando flexibilizar suas regras (Stengel, 2011). A presença de monitoramento parental, isto é, os pais terem conhecimento do comportamento dos filhos, seus amigos e os ambientes frequentados, está relacionado ao menor envolvimento em comportamentos de risco como comportamento infracional e uso de substâncias (Fosco et al., 2012). Também, o apoio dos professores se correlacionou negativamente com todos os comportamentos de risco. A compreensão de que esse apoio pode ser considerado como fator protetivo no envolvimento em riscos também foi evidenciada nos estudos de Costa (2009) e Wang e Dishion (2011). Além do apoio dos pais, é importante que os jovens possam contar com outros adultos e o apoio dos professores pode auxiliá-los nas mudanças ocorridas não só na adolescência como também no contexto escolar. No entanto, de acordo com Wang e Dishion (2011), durante a adolescência, os alunos têm muitos professores e pouco tempo de interação com eles, o que dificulta a aproximação e a criação de laços mais sólidos com os mesmos.

Quanto às diferenças por sexo encontradas na percepção de apoio social e no envolvimento em comportamentos de risco, verificou-se que os meninos apresentaram médias superiores às meninas no comportamento sexual de risco, no comportamento infracional e comportamento de risco total e as meninas apresentaram maiores médias em comportamento suicida. No que se refere à percepção de apoio social, as médias demonstram que as meninas percebem mais apoio dos amigos e no valor total da escala, além de apresentarem médias superiores aos meninos em todos os subtipos de apoio social, embora sem significância estatística. Esses dados revelam que há diferença de sexo na percepção de apoio social e envolvimento em comportamentos de risco. Pode-se compreender essa diferença a partir de uma concepção de gênero, definido como uma construção social e histórica, compreendendo que padrões de comportamentos fazem parte de uma construção em que a sociedade valida condutas como verdadeiras e esperadas (Colling, 2004). Discutir resultados a partir da perspectiva de gênero permite a desconstrução de padrões determinados como certos e estereotipados (Colling, 2004; Taquette, Vilhena, \& Paula, 2004).

$\mathrm{O}$ maior envolvimento de meninos em comportamento sexual de risco e comportamento infracional pode ser explicado devido a questões culturais e sociais, já que é esperado o envolvimento precoce dos meninos em atividades sexuais, valorização da hipersexualidade e da virilidade (Bordin \& Sperb, 2012; Springer et al., 2006; Taquette et al., 2004). Os jovens tendem a ser criados com maior liberdade pela família e apresentar mais condutas de enfrentamento violento, ligadas a atributos masculinos como a valentia (Câmara et al., 2007; Springer et al., 2006). Por outro lado, são esperados das meninas comportamentos associados à maior passividade, hipossexualidade, além de ter o primeiro intercurso sexual com namorados ou parceiros fixos, em geral, mais tarde do que os meninos (Bordini \& Sperb, 2012; Taquette et al., 2004).

No que se refere ao comportamento suicida ser mais prevalente entre as meninas, os estudos ressaltam que a ideação e tentativa de suicídio, além da presença de sintomas depressivos, são mais prevalentes entre as jovens enquanto que o suicídio propriamente dito ocorre mais entre os meninos que utilizam meios mais agressivos que, com frequência, levam ao êxito do suicídio (Abasse et al., 2009; Bahls \& Bahls, 2002; Braga \& Dell'Aglio, 2013). Esse resultado pode ser compreendido a partir dos estereótipos de gênero, expectativas sociais, diferenças do tipo de educação e socialização entre meninos e meninas. Em relação às meninas é socialmente esperado que sejam mais contidas e menos agressivas que os meninos, o que pode explicar a utilização de métodos mais agressivos entre os jovens do sexo masculino (Braga \& Dell'Aglio, 2013). Além disso, meninos e meninas tendem a enfrentar problemas de forma diferente, 
sendo que para as jovens, é permitido socialmente que extravasem suas emoções enquanto que os meninos tendem a apresentar comportamentos de evitação ao problema, que pode levar a uma sobrecarga emocional e ao alívio da tensão através de comportamentos de risco à sua saúde (Câmara \& Carlotto, 2007).

A diferença da percepção de apoio social dos amigos e no total da escala entre os adolescentes também pode ser discutida a partir de estereótipos de gênero já que é esperado que as meninas compartilhem seus problemas sendo que, geralmente, segundo Springer et al. (2006) são criadas mais próximas à família e com maior monitoramento parental. Por outro lado, os meninos são criados com maior liberdade em relação às interações sociais, sendo permitido que frequentem outros espaços além da escola e da família. Essas questões culturais também podem influenciar na percepção da disponibilidade de apoio social por parte dos meninos (Rueger et al., 2010). Logo, esses estereótipos de gênero parecem estar relacionados à manifestação dos comportamentos de risco e na percepção de apoio social, podendo explicar as diferenças encontradas neste estudo.

\section{CONSIDERAÇÕES FINAIS}

Este estudo apresentou algumas considerações ao avaliar simultaneamente quatro comportamentos de risco e ao verificar sua relação com o apoio social, sugerindo algumas reflexões. A variável idade destacou-se como influente no processo de manifestação dos comportamentos de risco durante a adolescência, demonstrando que a adolescência é um momento de experimentação e busca de novidades. No entanto, a experimentação não se mostrou uniforme, pois parece haver períodos de maior envolvimento em riscos durante a adolescência média e final, já que nesse momento os jovens tendem a estar mais próximos dos seus amigos e mais afastados do contexto familiar do que no início da adolescência. Nesse sentido, o apoio dos amigos foi evidenciado como um fator que pode influenciar na adesão aos comportamentos de risco já que o adolescente pode ser encorajado a envolver-se em algum risco considerando a pressão dos pares e o sistema de autorregulação que ainda está se desenvolvendo. Por conseguinte, o apoio da família e dos professores parece influenciar na direção contrária ao envolvimento em comportamentos de risco, demonstrando que o apoio nesses contextos pode contribuir para o sentimento de pertencimento e ligação afetiva.

O gênero também parece influente no engajamento em comportamentos de risco e na percepção de apoio social. As questões sociais e culturais parecem influenciar na maior aceitação de uma sexualidade proeminente em meninos, além de ser mais aceito que eles se envolvam em comportamentos como brigas com agressão física, pichação, entre outros. Já as meninas tendem a expressar mais suas emoções, o que pode contribuir no comportamento suicida. Também, o fato do apoio social ser mais percebido por meninas pode estar relacionado ao que é esperado, culturalmente, de que elas mantenham relações mais próximas com as fontes de apoio social. Porém, isso não significa que os meninos não percebem apoio social, mas que valorizam ou percebem de maneira diferente das meninas. Sendo assim, destaca-se a necessidade de intervenções que levem em consideração os ambientes de inserção dos jovens e, em especial, o apoio social advindo da família, amigos e professores, além das questões de gênero que podem influenciar na adesão aos comportamentos de risco.

Como limitações do estudo, ressalta-se a realização de duas etapas de coleta de dados, ocasionando uma perda amostral significativa. Para estudos futuros, sugere-se investigar a relação de comportamento de risco e apoio social em outros contextos, como, por exemplo, em adolescentes estudantes de escolas particulares e adolescentes expostos a situações de riscos. Ao investigar o apoio social, seria interessante considerar o apoio de outros membros do contexto escolar como funcionários e equipe diretiva da escola. Quanto aos comportamentos de risco, seria interessante conhecer o envolvimento dos jovens em outros comportamentos, como comportamento alimentar não saudável, falta ou excesso de atividades físicas, uso/abuso de meios eletrônicos e comportamento de risco no trânsito. Estudos longitudinais no contexto brasileiro poderiam ajudar a compreender o processo de engajamento em comportamentos de risco e sua relação com apoio social, considerando o início da adolescência até o início da idade adulta. Por conseguinte, destaca-se que conhecer a relação do apoio social e diferentes comportamentos de risco pode trazer contribuições importantes às políticas públicas destinadas ao público juvenil, favorecendo estratégias de prevenção e intervenção. 


\section{REFERÊNCIAS}

Abasse, M., Oliveira, R., Silva, T., \& Souza, E. (2009). Análise epidemiológica da morbimortalidade por suicídio entre adolescentes em Minas Gerais, Brasil. Ciência e Saúde Coletiva, 14(2), 407-416. http://dx.doi.org/10.1590/S141381232009000200010

Albert, D. \& Steinberg, L. (2011). Judgment and decision making in adolescence. Journal of Research on Adolescence, 21(1), 211-224. http://dx.doi.org/10.1111/j.1532-7795.2010.00724.x

Albert, D., Chein, J., \& Steinberg, L. (2013). The teenage brain: Peer influences on adolescent decision making. Current Direction in Psychological Science, 22, 114-120. http://dx.doi.org/10.1177/0963721412471347

Alves, C. F., Zappe, J. G., \& Dell'Aglio, D. D. (no prelo). Índice de comportamentos de risco (ICR): Construção e evidências de validade psicométrica. Estudos de Psicologia.

Amparo, D., Galvão, A., Cardenas, C., \& Koller, S. H. (2008). A escola e as perspectivas educacionais de jovens em situação de risco psicossocial: Perspectivas educacionais dos jovens. Revista Semestral da Associação Brasileira de Psicologia Escolar e Educacional, 12(1), 69-88.

Araújo, Y., Neusa, C., Gomes, I., \& Nóbrega, R. (2011). Enfrentamento do adolescente em condição crônica: Importância da rede social. Revista Brasileira de Enfermagem, 64(2), 281-286. http://dx.doi.org/10.1590/S0034-71672011000200010

Auerbach, R., Bigda-Peyton, J., Eberhart, N., Webb, C., \& Ringo Ho, M. (2011). Conceptualizing the prospective relationship between social support, stress, and depressive symptoms among adolescents. Journal of Abnormal Child Psychology, 39, http://dx.doi.org/10.1007/s10802-010-9479-x

Bahls, S. \& Bahls, F. (2002). Depressão na adolescência: Características clínicas. Interação em Psicologia, 6(1), 49-57.

Barbetta, P. A. (2001). Estatística aplicada às ciências sociais. Florianópolis: UFSC.

Bordini, G. \& Sperb, T. (2012). Concepções de gênero nas narrativas de adolescentes. Psicologia: Reflexão e Crítica, 25(4), 738-746. http://dx.doi.org/10.1590/S0102-79722012000400013

Braga, L. L. \& Dell'Aglio, D. D. (2013). Suicídio na adolescência: Fatores de risco, depressão e gênero. Contextos Clínicos, 6(1), 2-14. http://dx.doi.org/10.4013/ctc.2013.61.01

Brener, N., Kann, L., Shanklin, S, Kinchen, S., Eaton, D., Hawkins, J., \& Flint, K. (2013). Methodology of the youth risk behavior surveillance system-2013. Morbidity and Mortality Weekly Report, 62(1), 1-20.

Brookmeyer, K., Henrich, C., Cohen, G., \& Shahar, G. (2011). Israeli adolescents exposed to community and terror violence: The protective role of social support. Journal of Early Adolescence, 31(4), 577-603. http://dx.doi. org/10.1177/0272431610366247

Câmara, S. G. \& Carlotto, M. (2007). Coping e gênero em adolescentes. Psicologia em Estudo, 12(1), 87-93. http:// dx.doi.org/10.1590/S1413-73722007000100011

Câmara, S. G., Sarriera, J, C., \& Carlotto, M. S. (2007). Predictores de conductas sexuales de riesgo entre adolescentes. Interamerican Journal of Psychology, 41(2), 161-166.

Castro, M., Cunha, S., \& Souza, D. (2011). Comportamento de violência e fatores associados entre estudantes de Barra do Garças, MT. Revista de Saúde Pública, 45(6), 1054-1061. http://dx.doi.org/10.1590/S0034-89102011005000072

Centers for Disease Control and Prevention (2012). Youth Risk Behavior Surveillance System. Atlanta: MMWR.

Choo, H. \& Sim, T. (2010). Health risk behaviours of foreign-born adolescents in Singapore: Exploration of risk factors in an Asian context. British Journal of Social Work, 40, 2203-2222. http://dx.doi.org/10.1093/bjsw/bcp160

Cobb, S. (1976). Social support as a moderator of life stress. Psychosomatic Medicine, 38(5), 300-314. http://dx.doi. org/10.1097/00006842-197609000-00003

Costa, L. G. (2009). A rede de apoio social de jovens em situação de vulnerabilidade social e o uso de drogas. Dissertação de mestrado não publicada. Programa de Pós-Graduação em Psicologia. Universidade Federal do Rio Grande do Sul, RS.

Costa, M., Junior, G., Isolan, L., Acosta, J., Jarros, R., Blaya, C., et al. (2013). Association between anxiety symptoms and problematic alcohol use in adolescents. Trends in Psychiatry and Psychotherapy, 35(2), 106-110. http://dx.doi. org/10.1590/S2237-60892013000200003

Cruzeiro, A., Souza, L., Silva, R., Pinheiro, R., Rocha, C., \& Horta, B. (2010). Comportamento sexual de risco: Fatores associados ao número de parceiros sexuais e ao uso de preservativos em adolescentes. Ciência e Saúde Coletiva, 15(1), 1149-1158. http://dx.doi.org/10.1590/S1413-81232010000700023

Dumas, T., Ellis, W., \& Wolfe, D. (2012). Identity development as a buffer of adolescent risk behaviors in the context of peer group pressure and control. Journal of Adolescence, 35, 917-927. http://dx.doi.org/10.1016/j.adolescence. 2011.12.012

Ellis, A., Nixon, R., \& Williamson, P. (2009). The effects of social support and negative appraisals on acute stress symptoms and depression in children and adolescents. British Journal of Clinical Psychology, 48, 347-361. http:// dx.doi.org/10.1348/014466508X401894 
Farias Júnior, J., Nahas, M., Barros, M., Loch, M., Oliveira, E., De Bem, M., \& Lopes, A. (2009). Comportamento de risco à saúde em adolescentes no Sul do Brasil: prevalência e fatores associados. Revista Panamericana de Salud Publica, 25(4), 344-352. http://dx.doi.org/10.1590/S1020-49892009000400009

Fosco, G., Stormshak, E., Dishion, T., \& Winter, C. (2012). Family relationships and parental monitoring during middle school as predictors of early adolescent problem behavior. Journal of Clinical Child \& Adolescent Psychology, 41(2), 202-213. http://dx.doi.org/10.1080/15374416.2012.651989

Kim, Y., Moon, S., \& Kim, M. (2011). Physical and psychosocial predictors of adolescents' suicide behaviors. Child and Adolescent Social Work Journal, 28, 421-438. http://dx.doi.org/10.1007/s10560-011-0241-1

Lerner, R. M. \& Galambos, N. L. (1998). Adolescent development: challenges and opportunities for research, programs, and policies. Annual Reviews Psychology, 49, 413-446. http://dx.doi.org/10.1146/annurev.psych.49.1.413

Levandowski, D., Barth, B., Munhós, A., Rodde, W., Wendland, J. (2012). Apoio familiar e gestação na adolescência: Um estudo qualitativo com adolescentes do Vale dos Sinos/RS. Interamerican Journal of Psychology, 46, 297-306.

Locatelli, D., Martins, S., Moura, Y., Noto, A., Opaleye, E., \& Sanchez, Z. (2011). Social factors associated to binge drinking: A cross-sectional survey among Brazilian students in private high schools. BCM Public Health, 11, 201-210. http://dx.doi.org/10.1186/1471-2458-11-201

Males, M. (2010). Is jumping off the roof always a bad idea? A rejoinder on risk taking and the adolescent brain. Journal of Adolescent Research, 25(1), 48-63. http://dx.doi.org/10.1177/0743558409353780

Michael, K. \& Ben-Zur, H. (2007). Risk-taking among adolescents: Associations with social and affective factors. Journal of Adolescence, 30, 17-31. http://dx.doi.org/10.1016/j.adolescence.2005.03.009

Nardi, F., Cunha, S., Bizarro, L., \& Dell'Aglio, D. D. (2012). Drug use and antisocial behavior among adolescents attending public schools in Brazil. Trends Psychiatry Psychotherapy, 34(2), 80-86. http://dx.doi.org/10.1590/S223760892012000200006

Raffaelli, M., Koller, S. H., \& Cerqueira-Santos, E. (2012). Protective factors moderate between risk exposure and problem behaviour among low income Brazilian adolescents. Psychology and Antisocial Behaviour in Schools, 9, 74-92.

Rueger, S., Malecki, C., \& Demaray, M. (2010). Relationship between multiple sources of perceived social support and psychological and academic adjustment in early adolescence: Comparisons across gender. Journal of Youth Adolescence, 39, 47-61. http://dx.doi.org/10.1007/s10964-008-9368-6

Siqueira, A., Tubino, C., Schwarz, C., \& Dell'Aglio, D. D. (2009). Percepção das figuras parentais na rede de apoio de crianças e adolescentes institucionalizados. Arquivos Brasileiros de Psicologia, 61(1), 176-190.

Springer, A., Parcel, G., Baumler, E., \& Ross, M. (2006). Supportive social relationships and adolescent health risk behavior among secondary school students in El Salvador. Social Science \& Medicine, 62, 1628-1640. http://dx.doi. org/10.1016/j.socscimed.2005.08.018

Squassoni, C. (2009). Suporte social: Adaptação transcultural do Social Support Appraisals e desenvolvimento socioemocional de crianças e adolescentes. Dissertação (Mestrado em Educação Especial). Programa de PósGraduação em Educação Especial, Universidade Federal de São Carlos, São Carlos, São Paulo.

Squassoni, C. E. \& Matsukura, T. S. (2014). Adaptação transcultural da versão portuguesa do Social Support Appraisals para o Brasil. Psicologia: Reflexão e Crítica, 27(1), 1-10. http://dx.doi.org/10.1590/S0102-79722014000100009

Steinberg, L. (2004). Risk taking in adolescence: What changes, and why? Annals New Tork Academy of Sciences, 1021, 51-58. http://dx.doi.org/10.1196/annals.1308.005

Steinberg, L. (2007). Risk taking in adolescence: New perspectives from brain and behavioral science. Current Directions in Psychological Science, 16(2), 55-59. http://dx.doi.org/10.1111/j.1467-8721.2007.00475.x

Stengel, M. (2011). Discursos de pais e mães sobre a amizade em famílias com filhos adolescentes. Paidéia, 21(49), 217-225. http://dx.doi.org/10.1590/S0103-863X2011000200009

Taquette, S., Vilhena, M., \& Paula, M. (2004). Doenças sexualmente transmissíveis e gênero: Um estudo transversal com adolescentes no Rio de Janeiro. Cadernos de Saúde Pública, 20(1), 282-290. http://dx.doi.org/10.1590/S0102$311 X 2004000100046$

Vaux, A., Philips, J., Holly, L., Thompson, B., Williams, D., \& Stewart, D. (1986). The Social Support Appraisals (SSA) Scale: Studies of reliability and validity. American Journal of Community Psychology, 4, 195-220. http://dx.doi. org/10.1007/BF00911821

Wagner, A., Falcke, D., Silveira, L., \& Mosmann, C. (2002). A comunicação em famílias com filhos adolescentes. Psicologia em Estudo, 7(1), 75-80. http://dx.doi.org/10.1590/S1413-73722002000100010

Wang, M., \& Dishion, T. (2011). The trajectories of adolescents' perceptions of school climate, deviant peer affiliation, and behavioral problems during the middle school years. Journal of Research on Adolescence, 22(1), 40-53. http://dx.doi.org/10.1111/j.1532-7795.2011.00763.x

Wang, M., \& Eccles, J. (2012). Social support matters: Longitudinal effects of social support of three dimensions of school engagement from middle to high school. Child Development, 83(3), 877-895. http://dx.doi.org/10.1111/j.14678624.2012.01745.x 
Weber, S., Puskar, K., \& Ren, D. (2010). Relationships between depressive symptoms, perceived social support, selfesteem, e optimism in a sample of rural adolescents. Issues in Mental Health Nursing, 31, 584-588. http://dx.doi. org/10.3109/01612841003775061

White, H., \& Jackson, K. (2005). Social and psychological influences on emerging adult drinking behavior. Alcohol, Research and Health, 28(4), 182-190.

Willoughby, T., Good, M., Adachi, P., Hamza, C., \& Tavernier, R. (2013). Examining the link between adolescent brain development and risk taking from a social-developmental perspective. Brain and Cognition, 83, 315-323. http:// dx.doi.org/10.1016/j.bandc.2013.09.008

Nota:

Pesquisa realizada com apoio financeiro do CNPq e FAPERGS.

Autores:

CÁssia Ferrazza Alves - Psicóloga, Mestre em Psicologia e Doutoranda do Programa de Pós-Graduação em Psicologia da Universidade Federal do Rio Grande do Sul.

DéBora Dalbosco Dell'Aglio - Psicóloga, Mestre e Doutora em Psicologia, docente do Programa de Pós-Graduação em Psicologia da Universidade Federal do Rio Grande do Sul.

Endereço para correspondência:

Cássia Ferrazza Alves

Universidade Federal do Rio Grande do Sul

Instituto de Psicologia

Rua Ramiro Barcelos, 2600, sala 117

CEP 90035-003 Porto Alegre, RS, Brasil

E-mail: cassiaferrazza@gmail.com

Recebido em: 06.08.14

Aceito em: 10.03 .15 